\title{
Exploring QSARs of some benzenesulfonamides incorporating cyanoacrylamide moieties as a carbonic anhydrase inhibitors (specifically against tumor-associated isoforms IX and XII)
}

\author{
Ahmed M. Alafeefy, Hatem A. Abdel-Aziz, F. Carta, Claudiu T. Supuran, \\ Shilendra K. Pathak, Onkar Prasad \& Leena Sinha
}

To cite this article: Ahmed M. Alafeefy, Hatem A. Abdel-Aziz, F. Carta, Claudiu T. Supuran, Shilendra K. Pathak, Onkar Prasad \& Leena Sinha (2015) Exploring QSARs of some benzenesulfonamides incorporating cyanoacrylamide moieties as a carbonic anhydrase inhibitors (specifically against tumor-associated isoforms IX and XII), Journal of Enzyme Inhibition and Medicinal Chemistry, 30:4, 519-523, DOI: 10.3109/14756366.2014.948435

To link to this article: https://doi.org/10.3109/14756366.2014.948435

+ View supplementary material 준

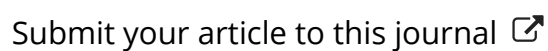

Published online: 08 Sep 2014.

Llll Article views: 203 


\title{
Exploring QSARs of some benzenesulfonamides incorporating cyanoacrylamide moieties as a carbonic anhydrase inhibitors (specifically against tumor-associated isoforms IX and XII)
}

\author{
Ahmed M. Alafeefy ${ }^{1}$, Hatem A. Abdel-Aziz ${ }^{2,3}$, F. Carta ${ }^{4,5}$, Claudiu T. Supuran ${ }^{4,5}$, Shilendra K. Pathak ${ }^{6}$, Onkar Prasad ${ }^{6}$, \\ and Leena Sinha ${ }^{6}$

\begin{abstract}
${ }^{1}$ Department of Pharmaceutical Chemistry, College of Pharmacy, Salman Bin Abdulaziz University, Alkharj, Saudi Arabia, ${ }^{2}$ Department of Research Center, Dokki, Cairo, Egypt, ${ }^{4}$ Department of Chemistry, Università degli Studi di Firenze, Polo Scientifico, Laboratorio di Chimica Bioinorganica, Via della Lastruccia 3, Sesto Fiorentino (Florence), Italy, ${ }^{5}$ Neurofarba Department, Section of Pharmaceutical and Nutriceutical
\end{abstract} \\ Pharmaceutical Chemistry, College of Pharmacy, King Saud University, Riyadh, Saudi Arabia, ${ }^{3}$ Department of Applied Organic Chemistry, National \\ Sciences, Università degli Studi di Firenze, Sesto Fiorentino (Florence), Italy, and ${ }^{6}$ Department of Physics, University of Lucknow, Lucknow, India
}

\begin{abstract}
Benzenesulfonamides incorporating cyanoacrylamide moieties with activity against tumour-associated human (h) isoforms hCA IX and XII (which are validated antitumor targets) were investigated for their quantitative structural activity relationships (QSAR). Multiple linear regression analysis was used to develop model relationships between molecular descriptors and inhibition constants $\left(\mathrm{K}_{\mathrm{i}}\right)$. The molecular geometry optimization were performed on all molecules at DFT-B3LYP/6-311++G(d,p) level. Over 1250 molecular descriptors were calculated using Gaussian 09, Hyperchem and EDRAGON programs. Multiple linear regression equations have been developed and validated using leave-one-out cross-validated technique. The derived QSAR models are found to be statistically significant and show good predictive ability.
\end{abstract}

\author{
Keywords \\ Benzenesulfonamides derivatives, carbonic \\ anhydrase isoforms hCA IX and XII, DFT, \\ MLR, QSAR descriptors
}

\section{History}

Received 8 June 2014

Revised 14 July 2014

Accepted 22 July 2014

Published online 3 September 2014

\section{Introduction}

Cancer is a major health problem which alarms the medical community all over the globe, and is projected to become the principal cause of death in the coming next few years ${ }^{1-4}$. In spite of the extensive progress in many aspects of cancer research, conventional chemotherapy is highly inadequate $e^{5-7}$. Newer drug entities with novel targets and mechanism of action are urgently needed $^{8-12}$. The tumor associated carbonic anhydrase (CA), especially CA IX and CA XII isoforms are known to be interesting tumor markers and their inhibitory action leads them to be promising antineoplastic agents, diagnostic tools for non-invasive in vivo tumor imaging and marking hypoxic tissues $^{13-17}$.

Recently, we have developed a new series of Tyrphostine- Type small molecules aiming to locate multiple tumor pathways inhibition to combat the multiple misregulated tumor growth and survival pathways ${ }^{18}$. In this respect, interesting derivatives with variable degrees of activity and affinity towards four CA isoforms were identified ${ }^{19}$ and work is in progress to look for their potential technological applications.

Address for correspondence: Leena Sinha, Department of Physics, University of Lucknow, Lucknow 226007, India Tel: +91 9415313779. E-mail: sinhaleena27@gmail.com
Computer aided drug design methodologies (CADD), such as quantitative structure-activity relationship (QSAR) and/ quantitative structure-property (QSPR) relationship, are undeniably prodigious quantum chemical studies of the modern day chemistry and biochemistry. QSAR/QSPR may help estimate biological activity data, cutting down cost and time consuming synthetic procedures, and predicting pharmacokinetic as well as pharmacodynamics profiles and ultimately constituting an important tool in design and development of new drug and novel leads. To achieve a convincing correlation, it is vital that only the relevant descriptors be used. Descriptors reflect simple molecular properties and in general can cater an insight into physicochemical attributes of activity ${ }^{20}$.

In our previous study, we had synthesized several benzenesulfonamides incorporating cyanoacrylamide moieties (tyrphostine analogues), and reported the new benzenesulfonamides obtained which were evaluated as the low nano-molar or sub-nanomolar CA IX/XII inhibitors ${ }^{19}$. The aim of present study is to find the main structural parameters of twenty-two benzenesulfonamides incorporating cyanoacrylamide moieties (Table 1) which are responsible for the inhibitory action against tumor associated carbonic anhydrase hCA IX/XII and to establish statistically significant mathematical models for their biological activities in terms the molecular descriptors. The results of this study may lead to synthesis of novel tumor associated isoforms hCA IX/XII inhibitor benzenesulfonamides. 
Table 1. Benzenesulfonamides incorporating cyanoacrylamide moieties used for QSAR analysis against hCA IX and XII.

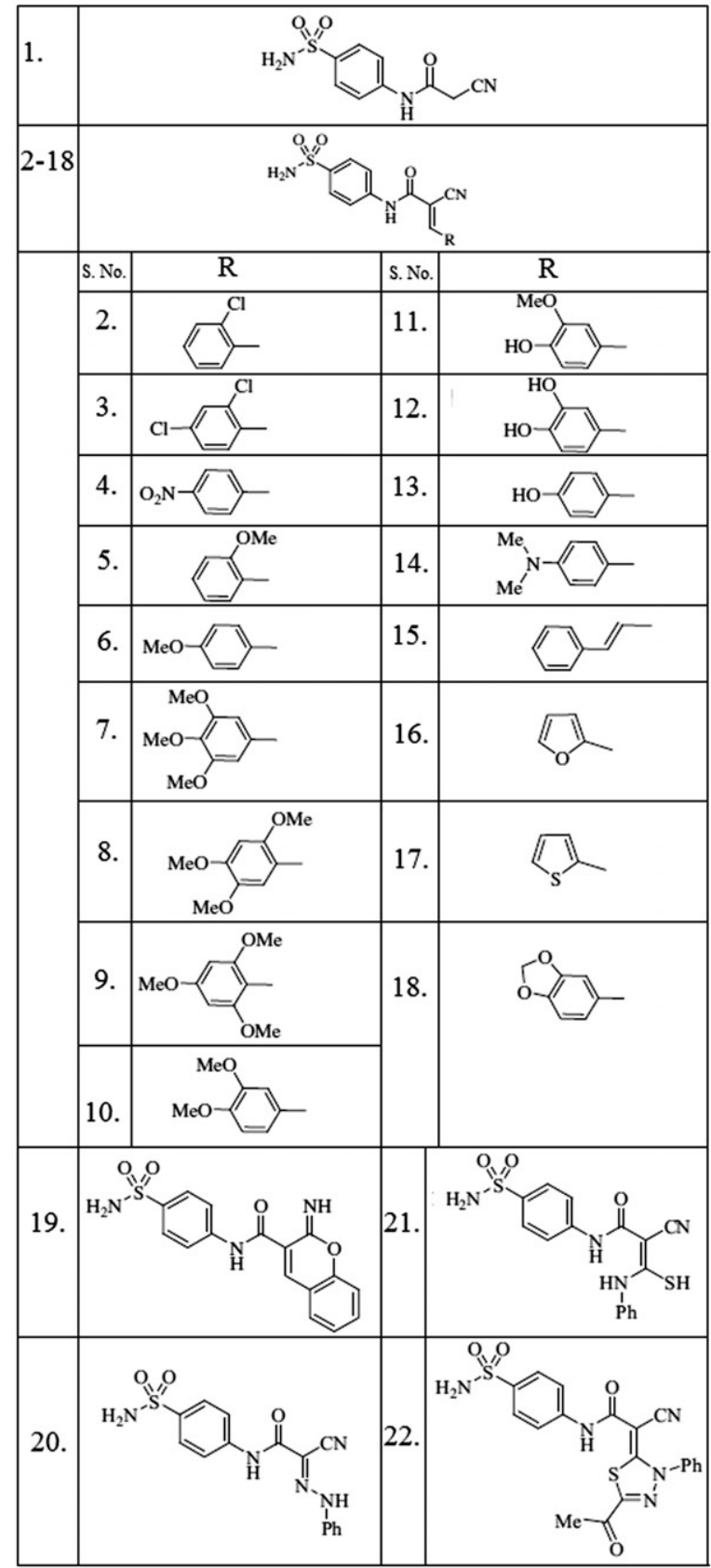

\section{Materials and methods}

\section{Experimental data set}

The present QSAR study is in continuation to our earlier work on benzenesulfonamide derivatives incorporating cyanoacrylamide moieties ${ }^{19}$. Which were synthesized for an action against tumor associated isoforms human carbonic anhydrase hCA IX and hCA XII. A dataset of 22 benzenesulfonamide derivatives presented in Table 2 was prepared and evaluated in terms of ability as a potent drug against tumor associated isoforms human carbonic anhydrase hCA IX and hCA XII.

\section{Geometry optimization and molecular descriptors calculation}

The initial conformations of the compounds have been drawn by means of the Gauss view 5.0.8 program $^{21}$ software and then 3D
Table 2. Experimental and predicted inhibition constants of benzenesulfonamides against human carbonic anhydrase (hCA) isoforms IX and XII along with residuals.

\begin{tabular}{|c|c|c|c|c|c|c|}
\hline \multirow[b]{3}{*}{$\begin{array}{l}\text { Mol. } \\
\text { S. No. }\end{array}$} & \multicolumn{6}{|c|}{$\mathrm{K}_{\mathrm{i}}(\mathrm{nM})$} \\
\hline & \multicolumn{3}{|c|}{ hCA IX } & \multicolumn{3}{|c|}{ hCA XII } \\
\hline & Exp. & $\begin{array}{c}\text { Pred. } \\
\text { (Equation 1) }\end{array}$ & Res. & Exp. & $\begin{array}{c}\text { Pred. } \\
\text { (Equation 2) }\end{array}$ & Res. \\
\hline 1 & 05.20 & 04.40 & 00.80 & 00.67 & 01.35 & -00.68 \\
\hline 2 & 08.90 & 09.70 & -00.80 & 00.86 & 00.11 & 00.75 \\
\hline 3 & 35.60 & 34.40 & 01.20 & 00.72 & 00.55 & 00.17 \\
\hline 4 & 00.93 & -01.60 & 02.53 & 00.66 & -00.41 & 01.07 \\
\hline 5 & 32.60 & 29.30 & 03.30 & 00.60 & 00.67 & -00.07 \\
\hline 6 & 00.86 & -00.90 & 01.76 & 00.57 & 00.50 & 00.07 \\
\hline 7 & 20.50 & 18.90 & 01.60 & 00.59 & 00.98 & -00.39 \\
\hline 8 & 00.96 & 01.30 & -00.34 & 00.67 & -00.23 & 00.90 \\
\hline 9 & 07.80 & 08.20 & -00.40 & 00.53 & 00.84 & -00.31 \\
\hline 10 & 21.20 & 25.10 & -03.90 & 02.30 & 01.29 & 01.01 \\
\hline 11 & 05.40 & 05.80 & -00.40 & 05.20 & 04.20 & 01.00 \\
\hline 12 & 00.65 & -00.40 & 01.05 & 02.30 & 02.09 & 00.21 \\
\hline 13 & 00.67 & 02.30 & -01.63 & 00.65 & 02.06 & -01.41 \\
\hline 14 & 03.40 & 06.20 & -02.80 & 00.93 & 01.86 & -00.93 \\
\hline 15 & 04.90 & 06.80 & -01.90 & 02.40 & 02.53 & -00.13 \\
\hline 16 & 04.30 & 05.80 & -01.50 & 06.30 & 06.00 & 00.30 \\
\hline 17 & 03.70 & 02.10 & 01.60 & 06.40 & 07.16 & -00.76 \\
\hline 18 & 09.50 & 05.90 & 03.60 & 08.90 & 09.23 & -00.33 \\
\hline 19 & 12.80 & 12.80 & 00.00 & 24.30 & 23.98 & 00.32 \\
\hline 20 & 09.50 & 10.70 & -01.20 & 03.50 & 03.71 & -00.21 \\
\hline 21 & 05.80 & 08.40 & -02.60 & 11.70 & 11.54 & 00.16 \\
\hline 22 & 00.33 & 00.30 & 00.03 & 00.64 & 01.38 & -00.74 \\
\hline
\end{tabular}

structures were optimized by employing Becke's three parameter hybrid exchange functional ${ }^{22}$ with Lee-Yang-Parr correlation functional (B3LYP) ${ }^{23,24}$ of $\mathrm{DFT}^{25}$ and using $6-311++\mathrm{G}(\mathrm{d}, \mathrm{p})$ basis set with the help of Gaussian 09 software $^{26}$ available at Central Facility for Computational Research (Cluster), University of Lucknow. After geometry optimization, several physiochemical parameters were calculated with Hyperchem software ${ }^{27}$.

A pool of molecular parameters (over 1250) including different group and class of 3D descriptors such as constitutional, walk and path counts, information indices, connectivity indices, topological, radial distribution function (RDF), 2D autocorrelation edge adjacency indices, Eigen value-based indices, topological charge indices, 3D-MoRSE, WHIM, GATAWAY, functional group counts, charge molecular properties, atom centered fragment and other group of descriptors have been calculated using, HYPERCHEM ${ }^{27}$ and EDRAGON ${ }^{28}$ software. We have also included five descriptors obtained from DFT calculation (total energy, energy of HOMO and LUMO, HOMO-LUMO gap, and molecular dipole moment) in the analysis.

\section{Statistical analysis}

In the present study, the statistical software package SPSS $21^{29}$ has been used for conducting Multiple linear regression (MLR) analysis. MLR analysis with stepwise selection and elimination of variables was employed to develop the best QSAR model. The MLR technique was performed on the set of molecules shown in Table 1. MLR analysis provides a useful equation based upon different quantum chemical descriptors that can be used to predict the $\mathrm{K}_{\mathrm{i}}$ of Benzenesulfonamide derivatives. Cross-validation technique with leave-one-out (loo) method $^{30}$ was used to verify the statistical significance and predictive ability of the developed model. 
Table 3. Cross-validation parameters.

\begin{tabular}{lccccccc}
\hline Model for & PRESS & SSY & PRESS/SSY & $S_{\text {loo-cv }}$ & $r_{\text {loo-cv }}^{2}$ & $r_{\text {adj }}^{2}$ & $r_{\text {adj }}^{2}-r_{\text {loo-cv }}^{2}$ \\
\hline hCA IX & 175.0 & 2108.0 & 0.083 & 2.886 & 0.917 & 0.961 & 0.044 \\
hCA XII & 021.4 & 0647.3 & 0.033 & 1.011 & 0.967 & 0.985 & 0.018 \\
\hline
\end{tabular}

\section{Results and discussion}

The chemical structure of the 22 compounds considered for the current study is given in Table 1 . The structural descriptors for the 22 benzenesulfonamides were computed by the procedure as explained in material and method section and the Pearson's correlation matrix was performed on all descriptors for selection of a set of appropriate descriptors. The analysis of the matrix revealed 12 descriptors for the development of MLR models. Step-wise multilinear regression analysis on these 12 molecular descriptors revealed the following two statistically significant independent parametric QSAR equations for biological activity (inhibition constant of title compounds against tumor associated human carbonic anhydrase isoform hCA IX and hCA XII) in terms of structural descriptors. Model 1 (for hCA IX)

$$
\begin{aligned}
\mathrm{K}_{\mathrm{i}}= & -180.578+448.427 \cdot \mathrm{E} 1 \mathrm{p}+143.313 \cdot \text { HATS7u } \\
& -171.384 \cdot \text { HATS7m }+2.977 \cdot \mathrm{RDF} 125 \mathrm{~m} \\
& -149.258 \cdot \mathrm{R} 8 \mathrm{p}-34.310 \cdot \text { GATS } 4 \mathrm{e}
\end{aligned}
$$

$\left(N=22, r=0.980, r^{2}=0.961, s=2.336, F=61.871, p<10^{-7}\right)$ where E1p is 1st component accessibility directional WHIM index, weighted by polarizability (WHIM descriptor), HATS7u leverage-weighted autocorrelation of lag 7/ un-weighted (GETAWAY descriptors), HATS7m - leverage-weighted autocorrelation of lag 7/weighted by mass (GETAWAY descriptors), RDF125m -Radial Distribution Function - 125/weighted by mass (RDF descriptors), R8p - R autocorrelation of lag 8/weighted by polarizability (GETAWAY descriptors), GATS4e - Geary autocorrelation of lag 4 weighted by Sanderson electronegativity (2D autocorrelations). The QSAR Model1 (Equation 1) suggests that the positive correlation coefficient of structural parameters E1p, HATS7u, RDF125m show a direct relationship with biological activity i.e. inhibition constant $\mathrm{K}_{\mathrm{i}}$. Parameters like HATS7m, R8p have negative correlation coefficient with inverse relationship for $\mathrm{K}_{\mathrm{i}}$. Model 2 (for hCA XII)

$$
\begin{aligned}
\mathrm{K}_{\mathrm{i}}= & 288.639-731.700 \cdot \mathrm{X} 1 \mathrm{~A}+259.252 \cdot \mathrm{Gu} \\
& -4.391 \cdot \mathrm{RDF030v}-14.385 \cdot \text { Mor08v } \\
& +37.907 \cdot \text { HATS6p }-41.660 \cdot \mathrm{G} 3 \mathrm{~m}
\end{aligned}
$$

$\left(N=22, r=0.992, r^{2}=0.985, s=0.804, F=106.28, p<10^{-7}\right)$ where $\mathrm{X} 1 \mathrm{~A}$ is average connectivity index of order 1 (Connectivity indices), $\mathrm{Gu}$ - total symmetry index/un-weighted (WHIM descriptors), RDF030v - Radial Distribution Function - 030/ weighted by van der Waals volume (RDF descriptors), Mor08vsignal 08/weighted by van der Waals volume (3D-MoRSE descriptors), HATS6p - leverage-weighted autocorrelation of lag 6/weighted by polarizability (GETAWAY descriptor) and G3m - 3rd component symmetry directional WHIM index/ weighted by mass (WHIM descriptors). The QSAR Model 2 for hCAXII indicates that the descriptors $\mathrm{Gu}$ and HATS6p have positive correlation coefficients whereas the remaining descriptors have negative correlation coefficients, implying the direct and inverse effect on the inhibition constant $\mathrm{K}_{\mathrm{i}}$ values respectively.

To check the credibility of the models, several parameters are used, such as regression coefficient $(r)$, adjusted square correlation coefficient $\left(r_{\text {adj }}^{2}\right)$, standard error of estimate $(S)$, value of Fischer's value $(F)$, significance level $(p)<0.005$. The large value of $F$, small value of $S$, even very small $p$ value, and $r^{2}$ close to 1 , all indicate the good predictability of the QSAR model. Both the developed QSAR models are statistically significant, the significance level being $(p)<10^{-7}$. The value of multiple correlation coefficient $(r)$ is found to be 0.980 and 0.992 for QSAR model 1 and 2 respectively and it supports the predictive/estimation ability of the both QSAR model (Equations 1 and 2).

The structural parameters used in Equations 1 and 2 are provided in Table S1 (supplementary material). Experimental and predicted values of the inhibition constant $\left(\mathrm{K}_{\mathrm{i}}\right)$ for both carbonic anhydrase inhibition are given here in Table 2 along with residuals.

Determination of predictive ability of the developed model needs its validation. To estimate the prediction ability of model by means of an internal validation procedure cross validation is conducted. In order to test the validity of the predictive power of selected MLR models (Equations 1 and 2), the leave-one-out technique ( $\mathrm{OOO}$ technique) was used. The developed models were validated by calculation of the following statistical parameters: predicted residual sum of squares (PRESS), total sum of squares deviation (SSY), standard deviation of predicted error and cross-validated correlation coefficients $\left(r_{\mathrm{cv}}^{2}\right.$ and $r_{\mathrm{adj}}^{2}$; Table 3).

PRESS also termed as $\mathrm{q}^{2}$ is an important cross-validation parameter as it is a good approximation of the real predictive error of the models. Its value being less than total sum of square (SSY) points out that model predicts better than chance and can be considered statistically significant. Smaller the PRESS value means the better of the model predictability. The results depicted in Table 3, confirm that the model 1 and 2 are statistically significant.

Further, for reasonable QSAR model, the PRESS/SSY ratio should be lower than 0.4. The ratio of PRESS/SSY in the present QSAR study are 0.083 and 0.033 for the first and second model, respectively. The predictive ability of both model is also corroborated by the leave-one-out cross validation, square correlation coefficient $\left(r_{\text {loo-cv }}^{2}=0.917\right.$ and 0.967 for model 1 and model 2 respectively) being very close to adjusted square correlation coefficient $\left(r_{\text {adj }}^{2}\right)$ for both the models. The agreement between the values of $r_{\text {loo-cv }}^{2}$ and $r_{\text {adj }}^{2}$ is an essential criterion for the best qualification of the QSAR models 1 and 2 .

Figures 1 and 2 show the plots of linear regression predicted values of inhibition constant $\left(\mathrm{K}_{\mathrm{i}}\right)$ versus the experimental $\mathrm{K}_{\mathrm{i}}$ values of the benzenesulfonamides incorporating cyanoacrylamide moieties with biological activity. The correlation plots between experimental and the predicted data from the derived multiple regression QSAR Equation 1 and 2 given in Figures 1 and 2 respectively, indicate that the predicted values are much closer to experimental one. This confirms that the models 1 and 2 can be successfully applied to predict inhibition constant of other benzenesulfonamides incorporating cyanoacrylamide moieties against tumour-associated human (h) isoforms hCA IX and XII.

\section{Conclusion}

Statistically significant QSAR models describing the structure carbonic anhydrase inhibition activity relationship in benzenesulfonamide derivatives incorporating cyanoacrylamide groups have been derived. The validity of the models has been 


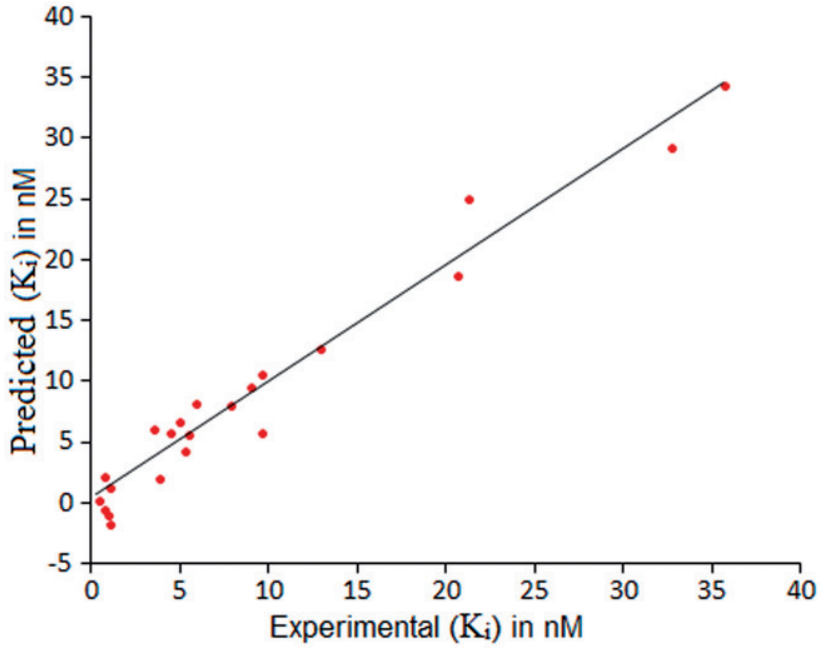

Figure 1. Plot of the predicted inhibitory constant for hCA IX against observed one using model 1.

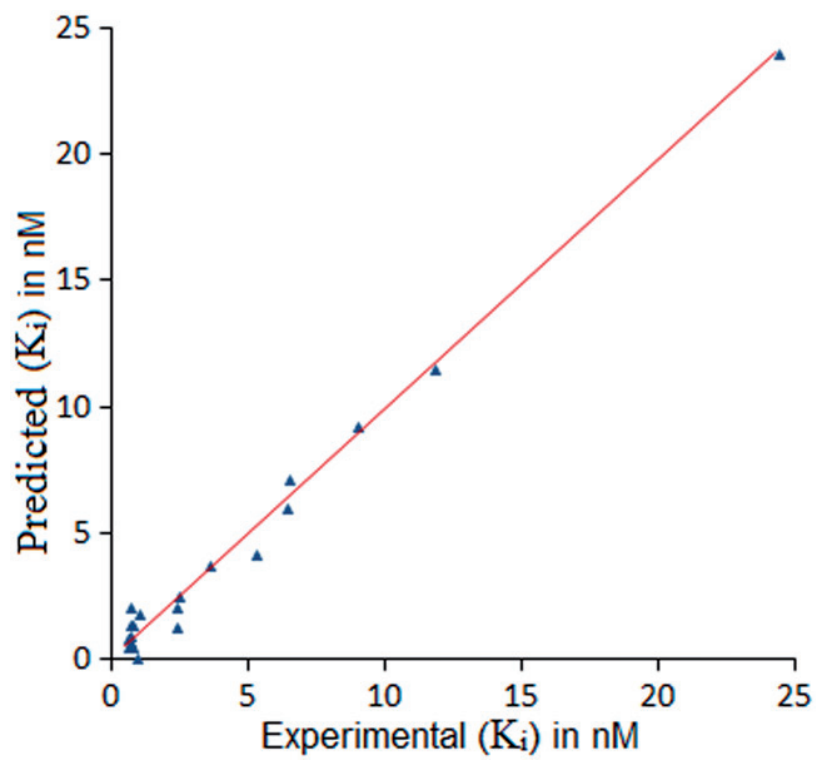

Figure 2. Plot of the predicted inhibitory constant for hCA XII against observed one using model 2.

established by the determination of suitable leave-one-out-cross validation (loo-cv) statistical parameters. The established models were used to predict the inhibition constant of the compounds under consideration and close agreement between experimental and predicted values was accomplished. The low-residual activity and large cross-validated values achieved suggests a decent predictive ability of the developed QSAR models. It indicates that the activity studied on benzenesulfonamide derivative series can be successfully modelled using various WHIM, GETAWAY, 3D-MoRSE, RDF and 2D autocorrelations molecular descriptors. The present study may lead to the synthesis of other potential tumour associated isoforms hCA IX/XII inhibitor benzenesulfonamide derivatives.

\section{Declaration of interest}

The authors report no conflicts of interest. One of the authors Shilendra K. Pathak is grateful to the University Grants Commission (UGC), New Delhi, India for the financial assistance as junior research fellowship.

\section{References}

1. Cope DG. Breast cancer: reviewing the past to give direction for the future. Oncol Nurs Forum 2013;40:425-8.

2. Jemal A, Bray F, Center MM, et al. Global cancer statistics. CA Cancer J Clin 2011;61:69-90.

3. DeSantis C, Virgo K, Stein K, et al. Cancer treatment and survivorship statistics 2012. CA Cancer J Clin 2012;62: 220-41.

4. Foye OW, Lemke LT, Williams AD. Principles of medicinal chemistry, 6th ed. Lippincott (PA): Williams and Wilkins; 2008.

5. Moeker J, Peat ST, Bornaghi LF, et al. Cyclic secondary sulfonamides: unusually good inhibitors of cancer-related carbonic anhydrase enzymes. J Med Chem 2014;57:3522-31.

6. Lehneck R, Neumann P, Vullo D, et al. Crystal structures of two tetrameric $\beta$-carbonic anhydrases from the filamentous Ascomycete sordariamacrospora. FEBS J 2014;281:1759-72.

7. Carroux JC, Rankin MG, Moeker J, et al. A prodrug approach toward cancer-related carbonic anhydrase inhibition. J Med Chem 2013;56:9623-34.

8. Rami M, Dubois L, Parvathaneni NK, et al. Hypoxia-targeting carbonic anhydrase IX inhibitors by a new series of nitroimidazolesulfonamides/sulfamides/sulfamates. J Med Chem 2013;56: 8512-20.

9. Monti MS, Supuran CT, De Simone G. Anticancer carbonic anhydrase inhibitors: a patent review (2008-2013). Expert Opin Ther Pat 2013;23:737-49.

10. Capasso C, Supuran CT. Anti-infective carbonic anhydrase inhibitors: a patent and literature review. Expert Opin Ther Pat 2013;23: 693-704.

11. Ward C, Langdon PS, Mullen P, et al. New strategies for targeting the hypoxic tumour microenvironment in breast cancer. Cancer Treat Rev 2013;39:171-9.

12. Supuran CT. Inhibition of carbonic anhydrase IX as a novel anticancer mechanism. World J Clin Oncol 2012;3:98-103.

13. Lee SH, Bae T, Lee HJ, et al. Rational drug repositioning guided by an integrated pharmacological network of protein, disease and drug. BMC Syst Biol 2012;2:6-80.

14. Garro Martinez JC, Vega-Hissi EG, Andrada MF, et al. Lacosamide derivatives with anticonvulsant activity as carbonic anhydrase inhibitors. molecular modeling, docking and QSAR analysis. Curr Comput Aided Drug Des 2014;10:160-7.

15. Malikowski MT, Bosch BJ, Min S, et al. Carbonic anhydrase inhibitors in corneal endothelial transport. Invest Ophthalmol Vis Sci 2014;55:2652-8.

16. Swenson ER. Safety of carbonic anhydrase inhibitors. Expert Opin Drug Saf 2014;13:459-72.

17. Gilmour MK. Perspectives on carbonic anhydrase. Comp Biochem Physiol A Mol Integr Physiol 2010;157:193-7.

18. Alafeefy MA, Alqasoumi IS, Ashour EA, et al. Quinazolinetyrphostin as a new class of antitumor agents, molecular properties prediction, synthesis and biological testing. Eur J Med Chem 2012; 53:133-40.

19. Alafeefy MA, Isik S, Abdel-Aziz HA, et al. Carbonic anhydrase inhibitors: benzenesulfonamides incorporating cyanoacrylamide moieties are low nanomolar/subnanomolar inhibitors of the tumorassociated isoforms IX and XII. Bioorg Med Chem 2013;21:1396403.

20. Wermuth GC. The practice of medicinal chemistry, 3rd ed. London: Academic Press; 2008:491-510.

21. Frisch A, Hratchian PH, Dennington II DR, et al. Gauss ViewVersion 5.0.8. Wallingford (CT): Gaussian, Inc.; 2009.

22. Beck DA. Density-functional thermochemistry. III. The role of exact exchange. J Chem Phys 1993;98:5648-52.

23. Lee C, Yang W, Parr GR. Development of the Colle-Salvetti correlation-energy formula into a functional of the electron density. Phys Rev B 1988;37:785-9.

24. Miehlich B, Savin A, Stoll H, Preuss H. Results obtained with the correlation-energy density functionals of Becke and Lee, Yang and Parr. Chem Phys Lett 1989;157:200-6.

25. Kohn W, Sham J. Self L-consistent equations including exchange and correlation effects. Phys Rev A 1965;140:1133-8.

26. Frisch JM, Trucks WG, Schlegel BH, et al. Gaussian 09W, Revision A.1. Wallingford (CT): Gaussian, Inc.; 2009. 
27. HyperChem (Molecular Modeling System) Hypercube, Inc, 1115 NW, 4th Street, Gainesville, FL 32601; USA, 2007. Available from: http://www.hyperchem.com/.

28. Tetko IV, Gasteiger J, Todeschini R, et al. Virtual computational chemistry laboratory design and description. J Comput Aid Mol Des 2005;19:453-63.
29. IBM Corp. Released 2012. IBM SPSS Statistics for Windows, Version 21.0. Armonk (NY): IBM Corp.

30. Hawkins MD, Basak CS, Mills D. Assessing model fit by crossvalidation. J Chem Inf Model 2003;43:579-86.

Supplementary material available online

Supplementary Table S1. 